\title{
AIRCRAFT POSITION DETERMINATION IN SBAS SYSTEM IN AIR TRANSPORT
}

\author{
Jaroslaw Kozuba $^{1}$, Kamil Krasuski ${ }^{2,}$, Janusz Cwiklak ${ }^{3}$, Henryk Jafernik ${ }^{3}$ \\ ${ }^{1}$ Silesian University of Technology, Poland; ${ }^{2}$ District Office in Ryki, Poland; \\ ${ }^{3}$ Polish Air Force Academy, Poland \\ aabuzok@wp.pl,kk_deblin@wp.pl,j.cwiklak@wsosp.pl, henryk21@interia.pl
}

\begin{abstract}
The article presents the results of GNSS satellite positioning in the SBAS support system in air transport. In accordance with ICAO recommendations, GNSS satellite technology may be implemented in air transport for GPS and GLONASS navigation systems as well as for their ABAS, SBAS and GBAS support segments. This academic article is intended to recreate the position of an aircraft using the SBAS support system, which is utilized in air transport. The position of the aircraft was determined using EGNOS differential corrections received from geostationary satellites, S120, S124, and S126. The mathematical model of the aircraft coordinate determination is based on absolute positioning (Single Point Positioning method - SPP). The research material comes from an in-flight experiment using a Cessna 172 aircraft, which was conducted at the military airfield in Dęblin, Poland. The computations of the Cessna 172 position were performed in the RTKLIB software (RTKPOST "Single" module). They were aimed at determining the coordinates of the Cessna 172 aircraft in the $X Y Z$ geocentric system. In order to analyze the test results obtained, a comparison was made with the coordinates of the Cessna 172 aircraft determined on basis of an EGNOS solution. The present work also specifies RMS errors of the Cessna 172 XYZ coordinates under comparison. The lowest dispersion for the difference in the $X Y Z$ coordinate values of the aircraft is based on the EGNOS S120 and S124 solutions. The greatest value of the RMS error for the X and Y aircraft coordinate difference occurs for the EGNOS S120 and S126 solutions. On the other hand, the greatest RMS error for the difference in the Z coordinate value of the aircraft may be seen for the comparison of the results of the EGNOS S124 and S126 solutions. Moreover, the article presents the verification and assessment of the ellipsoidal height h parameter for the Cessna 172 aircraft.
\end{abstract}

Keywords: GPS, air transport, SPP method, EGNOS, SBAS system.

\section{Introduction}

GNSS navigation systems and SBAS support systems are used in air transport [1]. Among the GNSS navigation systems, the GPS and GLONASS systems are certified to be used in aviation. The following geostationary satellite systems may be distinguished within the SBAS support system: WAAS, SDCM, GAGAN, MSAS, or EGNOS [2]. The European EGNOS system is used in civil aviation for the precise aircraft positioning in air transport. There are the following main objectives of the EGNOS implementation and use in air transport [3]:

- improvement of aircraft positioning accuracy for the SBAS system,

- providing information on the reliability of navigation data being transmitted to the user,

- synchronization of GPST time with UTC time,

- improvement of the availability, continuity, and reliability of satellite positioning in aviation,

- integration with GPS, GLONASS, and GALILEO navigation systems,

- EGNOS system application in geodetic, geophysical, and navigation calculations in real-time and post-processing mode,

- implementation of the EGNOS system for the SBAS APV landing procedures.

A great advantage of the EGNOS system is its practical application and implementation in the SBAS APV landing approach procedure. In Poland, the Polish Air Navigation Services Agency (PANSA), with headquarters in Warsaw, is responsible for implementing the operating procedures of the EGNOS system in air transport. It should be stressed that the EGNOS system in Poland will have a concrete implementation within the scope of the NPA GNSS approach procedure, and, particularly, SBAS APV-I [4]. In the non-precision GNSS NPA approach procedure, the assumed horizontal aircraft positioning accuracy may be up to $220 \mathrm{~m}$. Moreover, the limit of Horizontal Protection Levels (HPL) for lateral navigation (LNAV) can be up to $556 \mathrm{~m}$. It should be noted, however, that there are no accuracy and reliability standards of satellite positioning for vertical navigation (VNAV) in the NPA GNSS procedure. Additionally, in the GNSS NPA procedure the availability of the aircraft positioning navigation solution must exceed $99 \%$. In the GNSS NPA procedure, the time to alarm is 10 s and the continuity of the navigation solution may not be worse than $10^{-4}$ per hour [5]. 
APV approach with vertical guidance is a landing procedure, in which readings from the onboard instrument are used. The APV procedure does not meet the requirements of a precise landing approach with a decision height of no less than 250 feet and the runway visual range (RVR) no less than $600 \mathrm{~m}$. The APV procedure comprises 2 landing approach types: APV Baro (vertical guidance provided by a pressure altimeter coupled with the flight management system, FMS) and the APV SBAS (vertical guidance provided by an integrated GNSS/SBAS satellite system) [6]. Within the SBAS APV landing procedure, we may distinguish the SBAS APV-I and SBAS APV-II approaches. In the SBAS APV-I and APV-II approach procedures, the assumed horizontal positioning accuracy of the aircraft may amount to up to $16 \mathrm{~m}$. In the vertical plane, the aircraft positioning accuracy is $20 \mathrm{~m}$ for the SBAS APV-I procedure and $8 \mathrm{~m}$ for the SBAS APV-II, respectively. In the SBAS APV-I procedure the time to alarm is $10 \mathrm{~s}$ and in the SBAS APV II 6s respectively. The continuity of the navigation solution of the aircraft position may not be worse than $8 \cdot 10^{-6}$ per $15 \mathrm{~s}$ for both SBAS APV-I and APV-II. The availability of the navigation solution used in aircraft positioning must, in turn, exceed $99 \%$ in both those procedures. In the SBAS APV-I and APV-II approach procedures, the assumed reliability limit of the aircraft horizontal positioning may amount to up to $40 \mathrm{~m}$. In the vertical plane, the aircraft positioning reliability limit is $50 \mathrm{~m}$ for the SBAS APV-I procedure and $20 \mathrm{~m}$ for the SBAS APV-II, respectively [7].

The objective of this article is to use the EGNOS system for precise aircraft positioning within the SBAS support system in air transport. The position of the aircraft was determined in RTKLIB software for the SPP (Single Point Positioning) method. The calculations used EGNOS differential corrections from satellites S120, S124, and S126. The in-flight research test using a Cessna 172 aircraft was conducted at the military airfield in Dęblin, Poland.

\section{Materials and methods}

The mathematical model of the observation equation with the use of EGNOS correction data in the SPP method is described as follows [8]:

$$
L=\rho+c \cdot(d t r-d t s)+I o n+T r o p+R e l+S D C B_{L 1}+R D C B_{L 1}+M p+P R C,
$$

where $l$-pseudorange L1-C/A in GNSS system, $m$;

$\rho$ - geometric distance between satellite and receiver, $\mathrm{m}$;

$\rho=\left(\left(x-X_{G N S S}\right)^{2}+\left(y-Y_{G N S S}\right)^{2}+\left(z-Z_{G N S S}\right)^{2}\right)^{0.5}, \mathrm{~m} ;$

$(x, y, z)$ - aircraft position referenced to geocentric coordinates, $\mathrm{m}$;

$\left(X_{G N S S}, Y_{G N S S}, Z_{G N S S}\right)$ - satellite coordinates in GNSS system, $\mathrm{m}$;

$c$ - speed of light, $\mathrm{m} \cdot \mathrm{s}^{-1}$;

$d t r$ - receiver clock bias, $\mathrm{s}$;

$d t s$ - satellite clock bias, $\mathrm{s}$;

Ion - ionospheric delay, m;

Trop - tropospheric delay, m;

Rel-relativistic effect, m;

$S D C B_{L 1}$ - instrumental biases for satellites, referenced to the L1-C/A code, m;

$R D C B_{L 1}$ - instrumental biases for satellites, referenced to the L1-C/A code, m;

$M_{P}$ - multipath effect for pseudorange, $\mathrm{m}$;

$P R C$ - differential correction from the EGNOS system including long-term and fast corrections, as well as ionospheric and tropospheric corrections, $\mathrm{m}$.

The coordinates of the aircraft related to the geocentric $X Y Z$ system are determined on the basis of the observation equation (1) in a stochastic process. Usually, the least squares method is used for the aircraft positioning iteration process [9]. Furthermore, a covariance matrix and a weighting matrix of GNSS measurements are used for the stochastic processing of GNSS observations. A fast and longterm correction and, additionally, an ionospheric and tropospheric correction are applied as components of the EGNOS differential correction [10].

The SPP absolute positioning method from equation (1) was used to reproduce the coordinates of the Cessna 172 aircraft in the test. In order to do that, L1 C/A code GPS measurements were used that were obtained from a dual frequency Topcon HiperPro receiver installed onboard the aircraft. Moreover, the calculations used a GPS navigation message and EGNOS differential corrections 
received from the S120, S124, and S126 satellites. The research material comes from an in-flight experiment, which was conducted at the military airfield in Dęblin, in the Lublin Region, Poland. The calculations were aimed at recreating the actual flight trajectory of the Cessna 172 aircraft along the $X Y Z$ geocentric coordinate axes. The calculations were performed in the RTKLIB software (RTKPOST "Single" module). For the needs of the calculations, the "Single" module in the RTKPOST library was configured as follows [11]:

- GNSS system: GPS/SBAS system,

- source of PRC differential corrections: EGNOS system,

- file of RINEX type: RINEX 2.11,

- positioning mode: kinematic,

- computation mode: post-processing,

- positioning type: Single,

- source of precise orbits and clocks: GPS broadcast ephemeris + SBAS message,

- GPS observations: L1-C/A code observations,

- adjustment processing: applied,

- a priori standard deviation of code measurement: $1 \mathrm{~m}$,

- cut off elevation: $5^{\circ}$,

- interval of computation: $1 \mathrm{~s}$,

- maximum number of iterations: 5 .

- initial position of aircraft: based on RINEX file header,

- reference frame: WGS-84 frame,

- mathematical model of computations: least square estimation in sequential processing,

- output coordinates of aircraft: geocentric (XYZ) and geodetic (BLh) coordinates,

- reference time: GPS Time,

- ionospheric delay: SBAS grid model,

- relativistic effect: applied,

- Sagnac effect: applied,

- traveling time correction of pseudorange between satellite and receiver: applied,

- troposphere model: SBAS model,

- instrumental biases SDCB: only TGD applied,

- instrumental biases RDDB: not applied,

- multipath effect: not applied.

\section{Results and discussion}

Within the framework of the research test conducted, the reliability of the Cessna 172 aircraft coordinates determined was verified for the SPP positioning method. To this end, the coordinates obtained in the geocentric $X Y Z$ system of the Cessna 172 aircraft were compared. Moreover, in the comparative analysis, the value of the Cessna ellipsoidal height $\mathrm{h}$ in the BLh geodetic coordinate system was checked.

In the first comparative test, the aircraft $X Y Z$ coordinate values were verified and checked for the EGNOS solution. On the basis of that, the difference of the $X Y Z$ aircraft coordinate value was determined for the SPP method as follows [12]:

$$
\begin{aligned}
& d x 1=x_{S 120}-x_{S 124} ; d y 1=y_{S 120}-y_{S 124} ; d z 1=z_{S 120}-z_{S 124} ; \\
& d x 2=x_{S 120}-x_{S 126} ; d y 2=y_{S 120}-y_{S 126} ; d z 2=z_{S 120}-z_{S 126} ; \\
& d x 3=x_{S 124}-x_{S 126} ; d y 3=y_{S 124}-y_{S 126} ; d z 3=z_{S 124}-z_{S 126}
\end{aligned}
$$

where $x_{S 120}$ - coordinates of the aircraft along the $X$ axis determined using the EGNOS differential corrections from the S120 satellite, m;

$x_{S 124}$ - coordinates of the aircraft along the $X$ axis determined using the EGNOS differential corrections from the S124 satellite, m;

$x_{S 126}$ - coordinates of the aircraft along the $X$ axis determined using the EGNOS differential corrections from the S126 satellite, m; 
$y_{S 120}$ - coordinates of the aircraft along the $Y$ axis determined using the EGNOS differential corrections from the S120 satellite, m;

$y_{S 124}$ - coordinates of the aircraft along the $Y$ axis determined using the EGNOS differential corrections from the S124 satellite, m;

$y_{S 126}$ - coordinates of the aircraft along the $Y$ axis determined using the EGNOS differential corrections from the S126 satellite, m;

$z_{S 120}$ - coordinates of the aircraft along the $Z$ axis determined using the EGNOS differential corrections from the S120 satellite, m;

$z_{S 124}$ - coordinates of the aircraft along the $Z$ axis determined using the EGNOS differential corrections from the S124 satellite, m;

$z_{S 126}$ - coordinates of the aircraft along the $Z$ axis determined using the EGNOS differential corrections from the S126 satellite, $\mathrm{m}$.

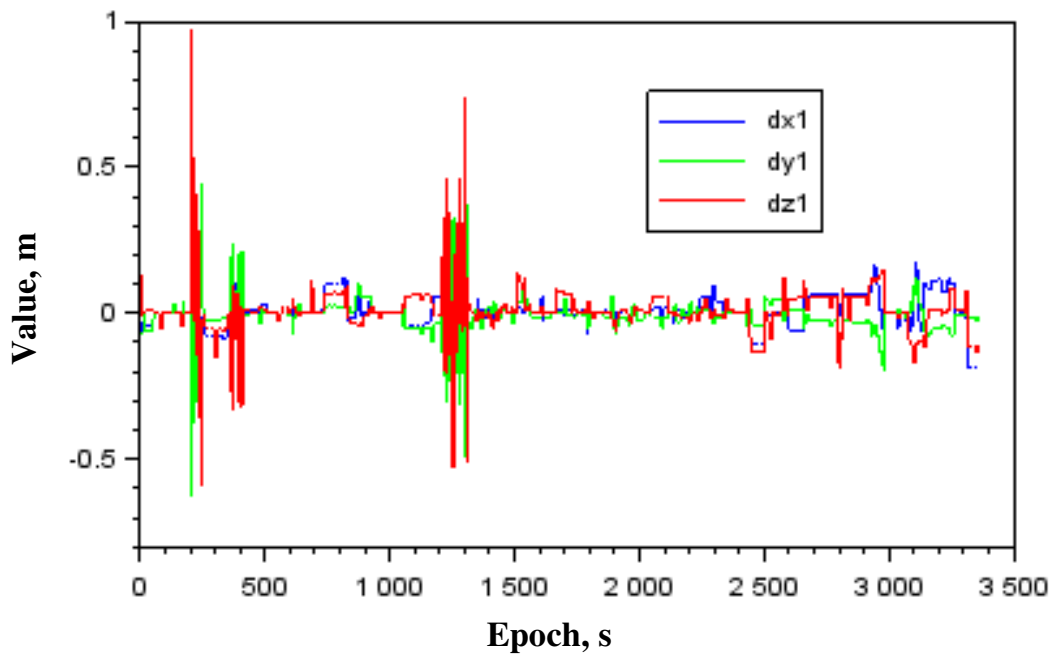

Fig. 1. Difference of aircraft $X Y Z$ coordinates based on EGNOS(S120) and EGNOS(S124) solutions

Figure 1 shows the results of comparing the $X Y Z$ coordinates of the aircraft based on EGNOS correction data received from satellites S120 and S124. Values of coordinate differences $(d x 1, d y 1$, $d z 1$ ) were determined on the basis of the equation (2). The mean $X$ coordinate difference value is 0.01 $\mathrm{m}$, and the RMS error [21] of this difference is $0.07 \mathrm{~m}$. The dispersion of the results for the $X$ coordinate difference is from $-0.39 \mathrm{~m}$ to $+0.71 \mathrm{~m}$. The mean $Y$ coordinate difference value is $-0.01 \mathrm{~m}$, and the RMS error [21] of this difference is $0.05 \mathrm{~m}$. Moreover, the amplitude of the results obtained for the $Y$ coordinate difference is from $-0.63 \mathrm{~m}$ to $+0.44 \mathrm{~m}$. The mean $Z$ coordinate difference value is $0.01 \mathrm{~m}$, and the RMS error [21] of this difference is $0.08 \mathrm{~m}$. Moreover, the amplitude of the results obtained for the $Z$ coordinate difference is from $-0.59 \mathrm{~m}$ to $+0.97 \mathrm{~m}$. It is worth noting that the span of the mean $(d x 1, d y 1, d z 1)$ parameter value is at the level of $\pm 0.01 \mathrm{~m}$. At the same time, the maximum level of the RMS error for the $(d x 1, d y 1, d z 1)$ parameter values reaches $0.1 \mathrm{~m}$. The dispersion of the results for all parameters $(\mathrm{dx} 1, \mathrm{dy} 1, \mathrm{dz} 1)$ is in the range of $-1 \mathrm{~m}$ to $+0.7 \mathrm{~m}$.

Figure 2 shows the results of comparing the $X Y Z$ coordinates of the aircraft based on EGNOS correction data received from satellites S120 and S126. Values of coordinate differences $(d x 2, d y 2$, $d z 2$ ) were determined on the basis of the equation (3). The mean value of the $d x 2$ parameter determined is $0.79 \mathrm{~m}$, and its RMS error is $1.05 \mathrm{~m}$. The dispersion of the results for the $d x 2$ parameter is from $-1.60 \mathrm{~m}$ to $+5.93 \mathrm{~m}$. The mean $d y 2$ parameter value is $-0.48 \mathrm{~m}$, and the RMS error of this parameter $0.49 \mathrm{~m}$. Moreover, the amplitude of the results obtained for the dy2 parameter is from $-2.05 \mathrm{~m}$ to $+1.09 \mathrm{~m}$. The mean $d z 2$ parameter value is $0.43 \mathrm{~m}$, and the RMS error $0.99 \mathrm{~m}$. Moreover, the amplitude of the results obtained for the $d z 2$ parameter is from $-2.91 \mathrm{~m}$ to $+4.13 \mathrm{~m}$. It is worth noting that the span of the mean $(d x 2, d y 2, d z 2)$ parameter value is at the level of $\pm 0.8 \mathrm{~m}$. At the same time, the maximum level of the RMS error for the $(d x 2, d y 2, d z 2)$ parameter values reaches $1.1 \mathrm{~m}$. The dispersion of the results for all parameters $(d x 2, d y 2, d z 2)$ is in the range of $-3 \mathrm{~m}$ to $+6 \mathrm{~m}$. 


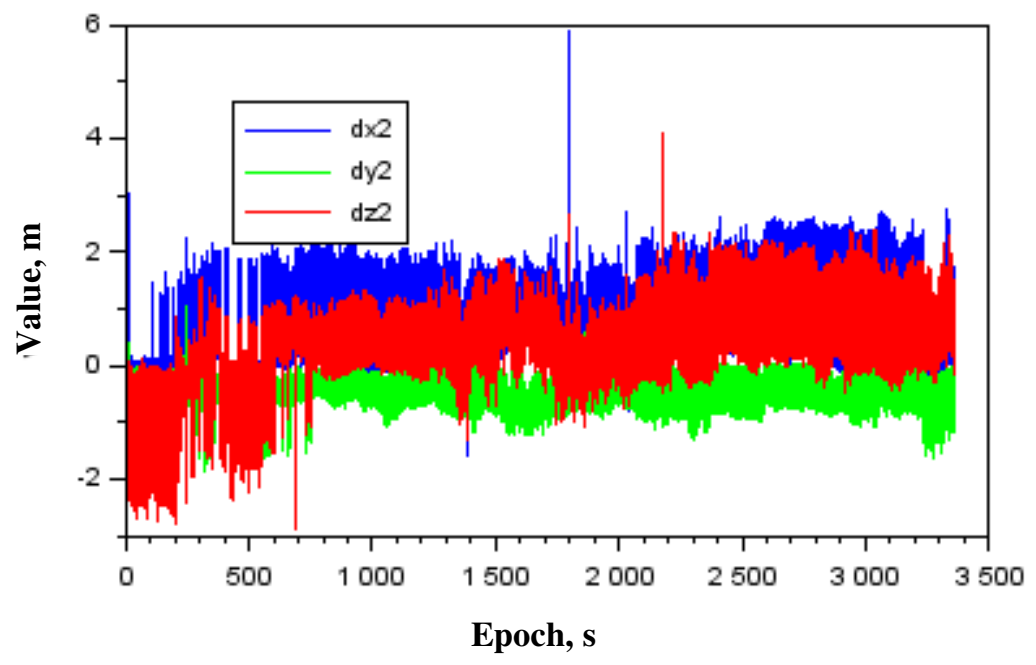

Fig. 2. Difference of aircraft $X Y Z$ coordinates based on EGNOS(S120) and EGNOS(S126) solutions

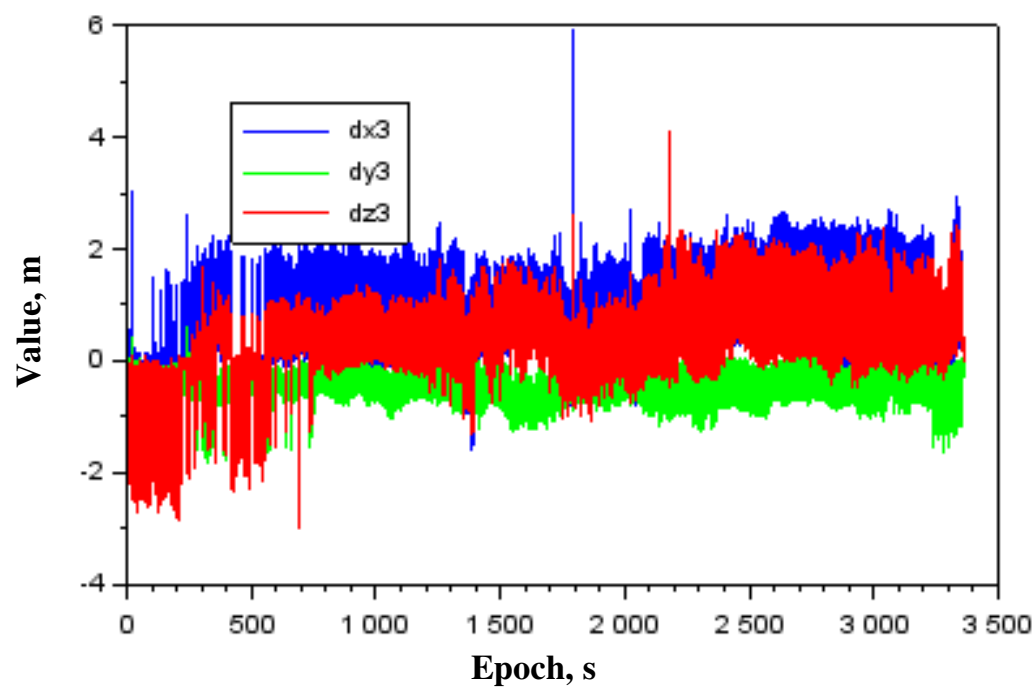

Fig. 3. Difference of aircraft $X Y Z$ coordinates based on EGNOS(S124) and EGNOS(S126) solutions

Figure 3 shows the results of comparing the $X Y Z$ coordinates of the aircraft based on EGNOS correction data received from satellites S124 and S126. Values of coordinate differences $(d x 3, d y 3$, $d z 3$ ) were determined on the basis of the equation (4). The mean value of the $d x 3$ parameter determined is $0.78 \mathrm{~m}$, and its RMS error is $1.04 \mathrm{~m}$. The dispersion of the results for the $d x 3$ parameter is from $-1.59 \mathrm{~m}$ to $+5.94 \mathrm{~m}$. The mean $d y 3$ parameter value is $-0.47 \mathrm{~m}$, and the RMS error of this parameter $0.48 \mathrm{~m}$. Moreover, the amplitude of the results obtained for the dy 3 parameter is from $1.87 \mathrm{~m}$ to $+1.10 \mathrm{~m}$. The mean dz3 parameter value is $0.42 \mathrm{~m}$, and the RMS error $1 \mathrm{~m}$. Moreover, the amplitude of the results obtained for the dz3 parameter is from $-3.02 \mathrm{~m}$ to $+4.15 \mathrm{~m}$. It is worth noting that the span of the mean $(d x 3, d y 3, d z 3)$ parameter value is at the level of $\pm 0.8 \mathrm{~m}$. At the same time, the maximum level of the RMS error for the $(d x 3, d y 3, d z 3)$ parameter values reaches $1.1 \mathrm{~m}$. The dispersion of the results for all parameters $(d x 3, d y 3, d z 3)$ is in the range of $-3.1 \mathrm{~m}$ to $+6 \mathrm{~m}$.

To sum up, the following conclusions may be drawn on the basis of the research results obtained:

- the lowest difference in the $X$ coordinate value of the aircraft is based on the EGNOS S120 and $\mathrm{S} 124$ solutions.

- the greatest RMS error for the difference in the $X$ coordinate value of the aircraft is seen for the comparison of the results of the EGNOS S120 and S126. 
- the lowest difference in the $Y$ coordinate value of the aircraft is based on the EGNOS S120 and $\mathrm{S} 124$ solutions.

- the greatest RMS error for the difference in the $Y$ coordinate value of the aircraft may be seen for the comparison of the results of the EGNOS S120 and S126 solutions.

- the lowest dispersion for the difference in the $Z$ coordinate value of the aircraft is based on the EGNOS S120 and S124 solutions.

- the greatest RMS error for the difference in the $Z$ coordinate value of the aircraft may be seen for the comparison of the results of the EGNOS S124 and S126 solutions.

The second test comprised a comparative analysis of the Cessna 172 ellipsoidal height $h$. Ellipsoidal height $\mathrm{h}$ was determined on the basis of the Helmert transformation in the iterative process [13]. In the calculations, the ellipsoidal height was determined separately using the EGNOS differential corrections received from the S120, S124, and S126 satellites. On the basis of that, the difference of the aircraft's ellipsoidal height value was determined for the SPP method as follows [12]:

$$
d h 1=h_{S 120}-h_{S 124} ; d h 2=h_{S 120}-h_{S 126} ; d h 3=h_{S 124}-h_{S 126},
$$

where $h_{S 120}$ - ellipsoidal height of the aircraft determined using the EGNOS differential corrections from the $\mathrm{S} 120$ satellite, $\mathrm{m}$;

$h_{S 124}$ - ellipsoidal height of the aircraft determined using the EGNOS differential corrections from the $\mathrm{S} 124$ satellite, $\mathrm{m}$;

$h_{S 126}$ - ellipsoidal height of the aircraft determined using the EGNOS differential corrections from the $\mathrm{S} 126$ satellite, $\mathrm{m}$.

Table 1

Results of ellipsoidal height comparisons for Cessna 172 aircraft

\begin{tabular}{|c|c|c|}
\hline Parameter & Value, m & RMS bias, m \\
\hline$d h 1$ & $\begin{array}{c}\text { The mean value of } d h 1 \text { equals } 0.01 \mathrm{~m} ; \\
\text { the dispersion of results for the } d h 1 \\
\text { parameter is between } \\
-0.59 \mathrm{~m} \text { and }+1.02 \mathrm{~m}\end{array}$ & $\begin{array}{c}\text { RMS bias amounts } \\
\text { to } 0.09 \mathrm{~m}\end{array}$ \\
\hline$d h 2$ & $\begin{array}{c}\text { The mean value of } d h 2 \text { equals } 0.68 \mathrm{~m} ; \\
\text { the dispersion of results for the } d h 2 \\
\text { parameter is between } \\
-3.30 \mathrm{~m} \text { and }+5.43 \mathrm{~m}\end{array}$ & $\begin{array}{c}\text { RMS bias amounts } \\
\text { to } 1.31 \mathrm{~m}\end{array}$ \\
\hline \multirow{2}{*}{$d h 3$} & $\begin{array}{c}\text { The mean value of } d h 3 \text { equals } 0.67 \mathrm{~m} ; \\
\text { the dispersion of results for the } d h 3 \\
\text { parameter is between } \\
-3.33 \text { m and }+5.42 \mathrm{~m}\end{array}$ & $\begin{array}{c}\text { RMS bias amounts } \\
\text { to } 1.32 \mathrm{~m}\end{array}$ \\
\hline
\end{tabular}

Table 1 presents a comparative analysis for the ellipsoidal height of the Cessna 172 aircraft for the EGNOS solution. The mean value of the $\mathrm{dh} 1$ parameter is $0.01 \mathrm{~m}$, while that of the $d h 2$ parameter is equal to exactly $0.68 \mathrm{~m}$, and $d h 3$ approximately $0.67 \mathrm{~m}$. The RMS error of the dh1 parameter is $0.09 \mathrm{~m}$, while that of the $d h 2$ parameter is equal to exactly $1.31 \mathrm{~m}$, and $d h 3$ approximately $1.32 \mathrm{~m}$. On the basis of the test results obtained, it can be concluded that the smallest difference in the aircraft's $h$ co-ordinate value is based on the EGNOS S120 and S124 solutions. On the other hand, the greatest RMS error for the difference in the $\mathrm{h}$ coordinate value of the aircraft may be seen for the comparison of the results received from the EGNOS S124 and S126 satellites.

\section{Conclusions}

The SBAS support system is increasingly widespread and utilized in air transport worldwide. In Poland, the SBAS support system will be used in the SBAS APV and NPA landing approach procedures. Therefore, the article presents the possibility of using the SBAS support system to determine the coordinates of an aircraft. To this end, the coordinates of the Cessna 172 aircraft were determined in the geocentric $X Y Z$ system. The calculations used EGNOS differential corrections from satellites S120, S124, and S126. The aircraft coordinates were determined in the RTKLIB software on the basis of the absolute positioning SPP method. Furthermore, GPS observation and navigation data 
were also used in the calculations. The work compares the XYZ coordinate values of the aircraft obtained from three independent solutions coming from the EGNOS S120, S124, and S126 satellites. Moreover, the article presents the verification of the ellipsoidal height h parameter for the Cessna 172 aircraft. On the basis of the results obtained, it can be concluded that similar values of the aircraft coordinates were obtained using EGNOS differential corrections received from the S120 and S124 satellites. On the other hand, the most significant discrepancies in the aircraft's coordinates were obtained by the application of EGNOS differential corrections received from the S126 satellite.

\section{References}

[1] Jaskievičová M., Džunda M., Satellite-based augmentation systems, Acta Avionica, Vol. XV, Number 27, 2013, pp. 1-6.

[2] Yun H., Kee C., Kim D., Korean Wide Are Differential Global Positioning System Development Status and Preliminary Test Results, International Journal of Aeronautical and Space Science, vol. 12 (3), 2011, pp. 274-282.

[3] European Commission Directorate-General for Energy and Transport, EGNOS Service Definition Document: Open Service, 2009.

[4] PANSA service website. [online] [01.01.2018]. Available at: http://www.pansa.pl/_site/index.php?menu_lewe=GNSS\&lang=_pl\&opis=wdrozenie.

[5] Grunwald G., Ciećko A., Bakuła M., Kaźmierczak R., Examination of GPS/EGNOS integrity in north-eastern Poland, IET Radar, Sonar \& Navigation, vol. 10 (1), 2016, pp. 114-121, doi: 10.1049/iet-rsn.2015.0053.

[6] Jafernik H., Krasuski K., Michta J., Assessment of suitability of radionavigation devices used in air, Scientific Journal of Silesian University of Technology. Series Transport, 2016, 90, pp. 99-112. ISSN: 0209-3324. DOI: 10.20858/sjsutst.2016.90.9. (in Polish)

[7] International Civil Aviation Organization. ICAO standards and recommended practices (SARPS), Annex 10 volume I (Radio navigation aids), 2006. [online] [01.01.2018]. Available at: http://www.ulc.gov.pl/pl/prawo/prawo-mi \%C4 \%99dzynarodowe/206-konwencje.

[8] Hernández-Pajares M., Miguel Juan J., Sanz J., Prats X., EGNOS Tutorial, gAGE Research Team, UPC, Catalonia, Spain. [online] [01.01.2018]. Available at: http://gage.upc.edu/sites/default/files/TEACHING_MATERIAL/SLIDES/EGNOS_tutorial_gAG E.pdf.

[9] Sanz Subirana J., Juan Zornoza J. M., Hernández-Pajares M., GNSS Data Processing, Volume I: Fundamentals and Algorithms, Publisher: ESA Communications, ESTEC, Noordwijk, Netherlands, ISBN 978-92-9221-886-7, 2013, pp. 139-145.

[10] Jimenez-Baňos D., Powe M., Matur A. R., Toran F., Flament D., Chatre E., EGNOS Open Service guidelines for receiver manufacturers", Proceedings of the 24th International Technical Meeting of The Satellite Division of the Institute of Navigation (ION GNSS 2011), 2011, pp. 2505-2512.

[11] Takasu T., RTKLIB ver. 2.4.2 Manual, RTKLIB: An Open Source Program Package for GNSS Positioning, 2013, pp. 29-49, [online] [01.01.2018] Available at: http://www.rtklib.com/prog/manual_2.4.2.pdf, 2013.

[12] Jaworski L, Świątek A., Zdunek R., EGNOS limitations over Central and Eastern Poland - results of preliminary tests EGNOS-EUPOS integration project, Artificial satellites, vol. 48, no. 3, 2013, pp. 93-102, DOI: 10.2478/arsa-2013-0008.

[13] Burtch R., A comparison of methods used in rectangular to geodetic coordinate transformations, Proceedings of the American Congress on Surveying and Mapping (ACSM) Annual Conference and Technology Exhibition, Orlando, FL, April 21-26, 2006, pp. 1-21. 